\title{
Editorial: Closing the Collection in Memory of Mildred S. Dresselhaus
}

It is well over a year now since Prof. Mildred S. Dresselhaus, a world-renowned condensed matter physicist, affectionately known as "Millie", passed away at the age of 86. To pay homage to her legacy, we dedicated the time span in 2018 from the anniversary of her passing in February to her birthday in November to a virtual issue of Physical Review Applied called "Millie Dresselhaus: Her living scientific legacy". With contributions on a wide range of topics that would have been of interest to Millie, we now close this collection in the present issue.

Scientists with a connection to Millie and her legacy have been invited by the editors of the "Millie issue" to contribute to the project. All articles have undergone the same strict peer review process as other contributions, to maintain the high quality standards of Physical Review Applied. We are very pleased with the result, and feel that even our small selection of 10 original articles, on topics from thermoelectrics to water desalination and metamaterials, illustrates clearly the progress in the scientific endeavor.

Some of the reported results provide a fresh perspective for understanding water diffusion in composites of carbon nanotubes and aromatic polyamide, thus allowing the optimization of such membranes for water desalination. We learned that understanding the formation of nanotube arrays and graphite intercalation compounds can be used to modify the morphology of these nanostructures for energy storage and charge transport. Similarly, uncovering a wet-chemical synthesis route to form $\mathrm{Bi}-\mathrm{Sb}$ thermoelectric nanowires opens the way to further increasing their thermopower at nanometer diameters. Thermal transport in graphene flakes can be optimized by changing their shape, and limiting the size of twodimensional silicon flakes may open the way to form $n$-type semiconductors with improved transport properties. Revealing a direct relationship between the figures of merit and power factors of semiconductors provides a way to optimize their performance, independent of shape and dimension. We learned that an optimum concentration of defects can improve photoluminescence efficiency in defective nanotubes, and that the magnetic behavior of nanographene-based microporous carbon may be tuned by changing the defect morphology. We also learned that arrays of graphene nanoribbons may form a metasurface capable of reshaping reflected waves. These findings form only the tip of the iceberg of progress in the field.

We believe that Millie would have been intrigued and inspired by all of these results. Readers may remember Millie typically sitting at or near the front row in a conference room, carefully taking notes and asking questions. This is where and how she received her inspiration for creating new science. Our wish is that this issue may be a similar source of inspiration for readers, as they endeavor to make their own contributions to science.

As Guest Editors, we hope to have succeeded in bringing together the scientific community, one more time, to commemorate the scientific legacy that Millie left behind. Finally, we wish to thank Dr. Julie Kim-Zajonz, Managing Editor of Physical Review Applied, for her inspiring idea to dedicate this commemorative issue to the scientific legacy of the late Prof. Mildred S. Dresselhaus.

Published 12 November 2018

Morinobu Endo

David Tománek

Guest Editors

Collection in memory of Mildred S. Dresselhaus

DOI: 10.1103/PhysRevApplied.10.050001 